\title{
Light-stimulated hydrogel actuators with incorporated graphene oxide for microfluidic applications
}

\author{
Lars Breuer ${ }^{1}$, Markus Raue ${ }^{2}$, Thomas Mang ${ }^{2}$, Michael J. Schöning ${ }^{1,3}$, \\ Ronald Thoelen ${ }^{4}$ und Torsten Wagner ${ }^{1,3}$ \\ ${ }^{1}$ Institute of Nano- and Biotechnologies (INB), FH Aachen, Germany \\ 2 Institute for Applied Polymer Chemistry (IAP), FH Aachen, Germany \\ ${ }^{3}$ Peter Grünberg Institute (PGI-8), Forschungszentrum Jülich GmbH, Germany \\ ${ }^{4}$ Institute for Materials Research (IMO), Hasselt University, Belgium \\ Contact: I.breuer@fh-aachen.de
}

\begin{abstract}
Summary:
Graphene oxide (GO) nanoparticles, which are able to convert light energy into heat, were incorporated into temperature-sensitive Poly-N-isopropylacrylamide (P-NIPAAm) hydrogels. In contrast to pure P-NIPAAm hydrogels, the new polymers with GO showed significant sensitivity towards light as a stimulus. The photothermal conversion and heating process of hydrogels under illumination were investigated by IRthermography. Furthermore, first experiments for a possible application as light-driven microvalves within lab-on-chip systems were performed.
\end{abstract}

Keywords: hydrogel, lab-on-chip systems, actuators, light-stimulation, graphene oxide, IR-thermography photopolymerization

\section{Introduction}

The interest in developing microfluidic devices for chemical and biological analysis has grown during the last years. These systems enable to work with smaller amounts of analyte samples and allow parallel operation leading to a significant decrease in operation time [1]. Furthermore, they give the ability to develop lab-on-chip systems, which combine different functions of a laboratory on a single chip [2].

Actuators such as valves, which control the flow through the device, are key components for the application of microfluidic systems. Often, conventional microvalves are smaller designs of their macroscopic counterparts and require expensive components as well as complex and cost intensive assembly [3]. Thus, new approaches and concepts for simpler and inexpensive actuators of disposable systems in the field of microfluidics are of great importance.

Because of their properties, hydrogels have become one of the most interesting material groups for these applications and are known as possible materials for micro-actuators. In general, hydrogels are polymer networks, which can swell in water and absorb large amounts of water without dissolving of the cross-linked polymer chains. Stimulus-sensitive hydrogels undergo significant volume changes in response to the variation of certain environmental parameters such as temperature, $\mathrm{pH}$ value and ion concentration, etc. [4].

In the past, temperature-triggered hydrogel valves based on Poly- $\mathrm{N}$-isopropylacrylamide (P-NIPAAm) were developed for possible applications in microfluidics and lab-on-chip systems [5,6]. The monomer of this hydrogel consists of both hydrophilic and hydrophobic side chains. Because of this, P-NIPAAm hydrogels show lower critical solution temperature (LCST) behavior: the hydrogel is swollen for temperatures below this critical value and undergoes a reversible collapse transition above the LCST $\left(32^{\circ} \mathrm{C}\right)$ so that the absorbed water diffuses out of the network [7]. However, conventional hydrogel valves require up to now an additional heating structure underneath the hydrogel layer which needs further fabrication and processing steps. To further lower the manufacturing costs, decrease complexity and increase flexibility, this work proposes the laterally resolved stimulation of hydrogel-based materials by a light source instead of a heating structure making these complex supporting structures obsolete. Furthermore, light as stimulus can be controlled temporarily as well as spatially with a high resolution and it can be used for contactless handling, which is beneficial for the sterile working environment in the field of cell culturing. Pure P-NIPAAm hydrogels are transparent materials 
with very low light absorption, which makes them useful for optical components such as adaptive liquid microlenses [8]. To increase the light absorption and to increase the sensitivity to light, temperature-sensitive hydrogels based on $\mathrm{P}$ NIPAAm were modified by incorporation of graphene oxide (GO) nanoparticles. These nanoparticles have an enhanced light absorption in the visible range and can convert photon energy into heat effectively [9]. Thus, hydrogels with GO can be heated above their transition temperature by illumination causing a light-driven collapse.

\section{Materials and fabrication methods}

N-isopropylacrylamide (NIPAAm, 99\%, SigmaAldrich), N,N'-methylenbisacrylamide (MBAm, 98\%, Merck) as crosslinker and 2-hydroxy-4-(2hydroxyethoxy)-2-methylpropiophenone (Irgacure 2959, Sigma-Aldrich) as photoinitiator were used without any further purification steps. The hydrogels were synthesized from a monomer solution with $100 \mathrm{mmol}$ monomer (NIPAAm), $1 \mathrm{mmol}$ crosslinker (MBAm) and $0.45 \mathrm{mmol}$ photoinitiator (Irgacure 2959) dissolved in $60 \mathrm{ml}$ deionized (DI) water.

The GO dispersions were prepared using a modified Hummers method [10]. For this purpose, $5 \mathrm{~g}$ graphite powder and $2.5 \mathrm{~g} \mathrm{NaNO}_{3}$ (Merck) were mixed in $120 \mathrm{ml}$ of $90 \%$ sulfuric acid $\left(\mathrm{H}_{2} \mathrm{SO}_{4}\right.$; VWR). $15 \mathrm{~g} \mathrm{KMnO} 4$ (98\%; Acros-Organics,) were added with a low addition rate to ensure a reaction temperature below $20^{\circ} \mathrm{C}$. Furthermore, the mixture was kept in an ice bath for cooling during this process step. After removing from the ice bath, the suspension was stirred for $24 \mathrm{~h}$ and $150 \mathrm{ml}$ of DI water were slowly stirred into the mixture followed by additional $24 \mathrm{~h}$ of stirring. After that, $50 \mathrm{ml}$ hydrogen peroxide $\left(\mathrm{H}_{2} \mathrm{O}_{2}, 30 \%\right.$; Merck) were added to the suspension. At the end, the mixture was washed with isopropanol and DI water for purification and treated in an ultrasonic bath for $4 \mathrm{~h}$ for exfoliation.

The hydrogel films were synthesized using a photopolymerization technique as described in [11]. For illumination with UV-light, a standard exposure unit for lithography processes (MJB 3; Süss MicroTec) was used which had an intensity of $10 \mathrm{~mW} / \mathrm{cm}^{2}$ at a wavelength of $365 \mathrm{~nm}$. For hydrogels with $\mathrm{GO}$, the pre-polymer solution was mixed with $\mathrm{GO}$ dispersions in a ratio of $1: 1$. For comparison, pure PNIPAAm gels were polymerized with a pre-polymer solution mixed with DI water in the same ratio. After the polymerization process, the hydrogels were kept in DI water for several days to remove non-polymerized toxic residuals of photoinitiator, cross-linker and monomer.

\section{Investigation of the photothermal heating process}

To investigate the conversion rate from photon energy into thermal energy and the influence of different GO concentrations, hydrogels with and without GO within the polymer network were illuminated with a blue laser diode (ULV-445-300; Roithner Lasertechnik). The power of the laser was $100 \mathrm{~mW}$ at a peak wavelength of $445 \mathrm{~nm}$. The dimensions of the hydrogels were $1 \mathrm{~cm} \times 2 \mathrm{~cm}$ and therefore much bigger than the diameter of the laser beam $(\sim 1 \mathrm{~mm})$ so that a spatially resolved stimulation of the hydrogels could be investigated (Fig. 1). The temperature distribution of the hydrogels under illumination was recorded with an

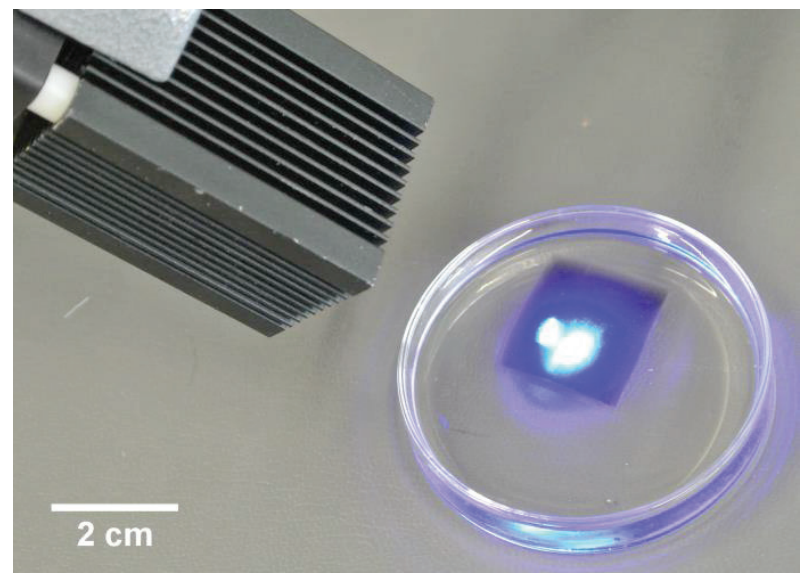

Figure 1: Setup for the investigation of the photothermal stimulation process of hydrogels with and without GO.

IR-camera (FLIR A5; FLIR Comercial Systems). An emissivity of $\varepsilon=0.95$ was assumed for the measurement and the hydrogels were placed on glass substrates to avoid undesired absorptions within the substrate. Figure 2 depicts two exemplary thermograms of a hydrogel with a GO concentration of $0.25 \mathrm{mg} / \mathrm{ml}$ obtained before illumination with the laser source (Fig. 2a) and after $120 \mathrm{~s}$ of illumination (Fig. 2b). At the beginning, the hydrogel shows a homogenous temperature distribution. When the laser source irradiates a hydrogel with GO, the GO nanoparticles absorb the incoming photons and convert their energy into heat. This leads to a heating process of the light-exposed part of the polymer and the hydrogel collapses when the LCST is exceeded. The maximum temperature difference between the hottest spot in the center of the laser beam and the remaining unilluminated hydrogel was measured to be $55^{\circ} \mathrm{C}$ after $120 \mathrm{~s}$ exposure for $100 \mathrm{~mW}$ laser power. Only areas under illumination are heated up facilitating the spatially resolved stimulation of certain parts of the 

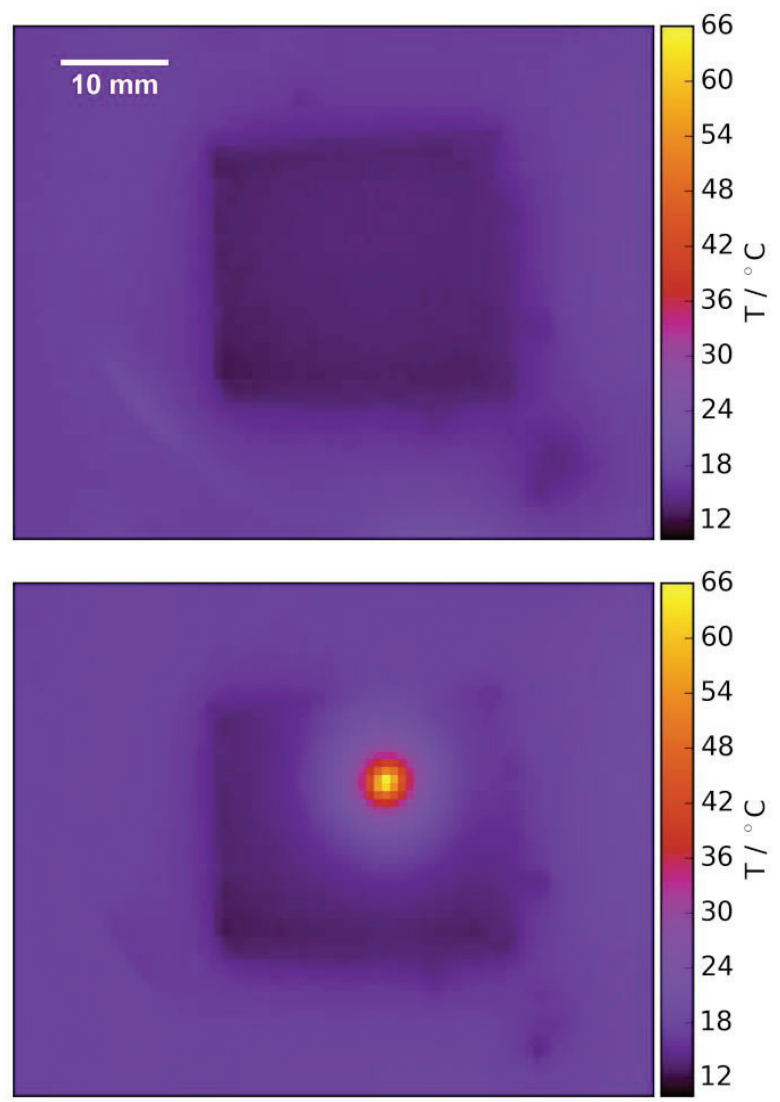

Figure 2: Thermograms of a hydrogel with a GO concentration of $0.25 \mathrm{mg} / \mathrm{ml}$ before (a) and after $120 \mathrm{~s}$ illumination (b) with a blue laser $(100 \mathrm{~mW}$, $445 \mathrm{~nm})$.

hydrogel. The temperature increase is proportional to the absorption coefficient of the hydrogel and raises with increasing GO concentration. For pure hydrogels without $\mathrm{GO}$ no significant temperature increase bigger than $2{ }^{\circ} \mathrm{C}$ occurred after $120 \mathrm{~s}$ demonstrating that the photothermal conversion process is done by the GO within the network.

\section{Flow control with microvalves in microfluidic systems}

To give a proof-of-concept for a possible application as microvalve, P-NIPAAm hydrogels containing $2 \mathrm{mg} / \mathrm{ml}$ of $\mathrm{GO}$ were fabricated in situ into microfluidic channels with dimensions of $0.4 \mathrm{~mm} \times 17 \mathrm{~mm} \times 3.8 \mathrm{~mm}$ (sticky-Slide Vl; Ibidi) as it is exemplarily depicted in figure 3 . The mass flow through the system was detected for different illumination states while a piezo pump (mp6; Bartels Mikrotechnik) was pumping water through the system at room temperature. When the light source was turned on, the hydrogel was heated above its transition temperature due to the light absorption of GO nanoparticles and collapsed. Thus, the cross section of the channel was no longer blocked by the hydrogel because the volume of the polymer network is much compared to the swollen state. This corresponds to the "open" status of the microvalve and a mass flow occurred through the microfluidic channel (Fig. 4). When the laser was turned off after 20 min., the hydrogel cooled down again, re-swelled and blocked the flow because of its increased volume in the channel. By fitting a sigmoidal function according to equation (1) to the data a mass flow of $\dot{\mathrm{m}}_{\text {collapsed }}=8.3 \pm 0.1 \mathrm{mg} / \mathrm{s}$ was determined. This flow reduces $6 \mathrm{~min}$. after the light source was turned to a rate of $\dot{\mathrm{m}}_{\text {swollen }}=0.7 \pm 0.2 \mathrm{mg} / \mathrm{s}$ off; the

$$
\dot{m}=\dot{m}_{\text {collapsed }}+\frac{\dot{m}_{\text {swollen }}-\dot{m}_{\text {collapsed }}}{1+\exp \left(\frac{t-t_{c}}{\Delta t}\right)}
$$

hydrogel re-swelled again so that the channel is nearly completely blocked. This demonstrates the ability of the P-NIPAAm hydrogel with GO to act as light-stimulated valve within microfluidic channels. Because the re-swelling process is limited by diffusion, the response time for the closing process is quite high for the macroscopic hydrogels used for these first experiments. By decreasing the hydrogel size and optimizing the geometry and design, it should be possible to improve the response time and to enable complete closing of the channel. Compared to hydrogel valves with a heating element beneath, it is beneficial that the heating process has a higher spatial resolution. The re-swelling process, which is slower than the collapse limits performance. In the case of lightstimulated hydrogels, this re-swelling starts earlier because the hydrogel cools down faster after the stimulus has been turned off.

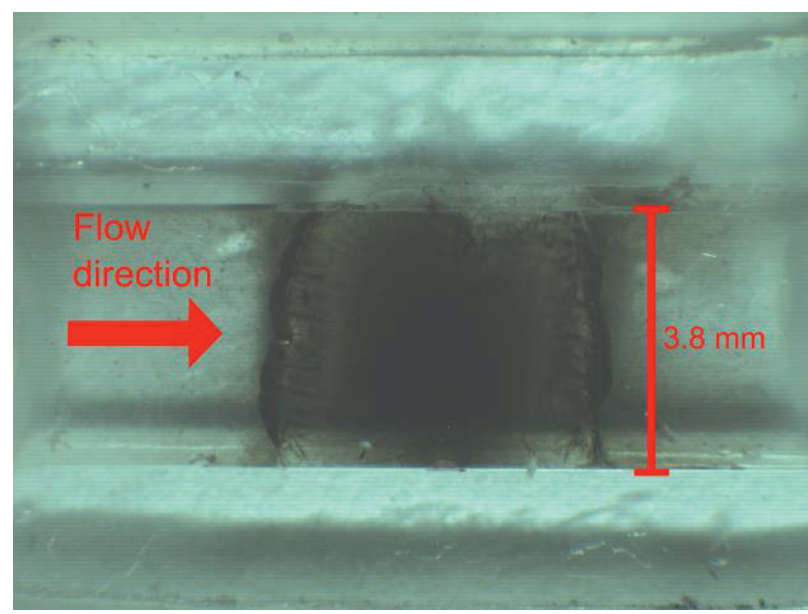

Figure 3: Photopolymerized hydrogel with GO within a microfluidic channel (sticky-Slide VI, lbidi). 


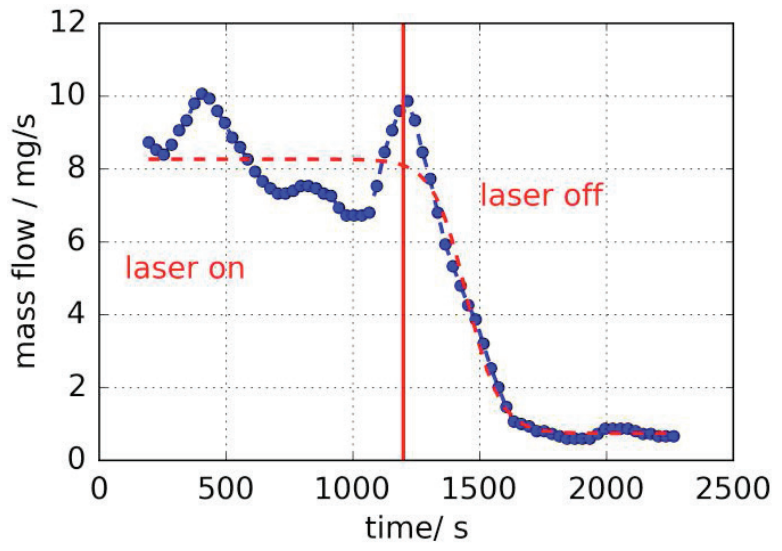

Figure 4: Mass flow through the microfluidic system for an "open" (laser on) and a "closed" hydrogel valve (laser off).

\section{Conclusion and outlook}

In this work, P-NIPAAm hydrogels with incorporated GO nanoparticles are presented. These nanoparticles are able to absorb light energy and convert it into heat effectively. In contrast to pure P-NIPAAm, hydrogels with GO could be heated up locally with a focused laser beam as could be demonstrated by IRthermography. Furthermore, a proof-of-concept for a possible application as light-stimulated valve within microfluidic systems was performed. Because the transition of the hydrogels is a diffusion process, the response time and leakage flow should be improved for future applications by further reduction of the size of the hydrogel actuator and optimization of the geometry.

\section{Acknowledgment}

This research project was funded by the German Federal Ministry of Education and Research (BMBF) within the research frame of "NanoMatFutur": 13N12585.

\section{Literature}

[1] BEEBE, D. J. ; MENSING, G. A. ; WALKER, G. M.: Physics and applications of microfluidics in biology. Annu. Rev. Biomed. Eng. 4 (2002), pp. 261-286; DOI: 10.1146/annurev.bioeng.4.112601.125916
[2] STONE, H. A. ; STROOCK, A. D. ; AJDARI, A. Engineering flows in small devices: microfluidics toward a Lab-on-a-Chip. Annu. Rev. Fluid Mech. 36 (2004), pp. 381-411; DOI: 10.1146/annurev.fluid.36.050802.122124

[3] WANG, J. ; CHEN, Z. ; MAUK, M. ; HONG, K.-S. ; LI, M. ; YANG, S. ; BAU, H. H.: Self-actuated, thermoresponsive hydrogel valves for lab on a chip. Biomed. Microdevices 7 (2005), pp. 313-322; DOI: 10.1007/s10544-005-6073-z

[4] QIU, Y. ; PARK, K.: Environment-sensitive hydrogels for drug delivery. Adv. Drug Deliv. Rev. 53 (2001), pp. 321-339; DOI: 10.1016/j.addr.2012.09.024

[5] RICHTER, A. ; KUCKLING, D. ; HOWITZ, S. ; GEHRING, T. ; ARNDT, K.-F.: Electronically controllable microvalves based on smart hydrogels: magnitudes and potential applications. $J$. Microelectromech. Syst. 12 (2003), pp. 748-753; DOI: 10.1109/JMEMS.2003.817898

[6] BÄCKER, M. ; RAUE, M. ; SCHUSSER, S. ; JEITNER, C. ; BREUER, L. ; WAGNER, P. POGHOSSIAN, A. ; FÖRSTER, A. ; MANG, T. ; SCHÖNING, M. J.: Microfluidic chip with integrated microvalves based on temperature- and $\mathrm{pH}$ responsive thin films. Phys. Status Solidi A 209 (2012), pp. 839-845; DOI: 10.1002/pssa.201100763

[7] KUCKLING, D. ; HARMON, M. E. ; FRANK, C. W.: Photo-cross-linkable PNIPAAm copolymers: 1. Synthesis and characterization of constrained temperature-responsive hydrogel layers. Macromolecules 35 (2002), pp. 6377-6383; DOI: $10.1021 / \mathrm{ma0203041}$

[8] KIM, J. ; SERPE, M. J. ; LYON, L. A.: Hydrogel microparticles as dynamically tunable microlenses. $J$. Am. Chem. Soc. 126 (2004), pp. 9512-9513; DOI: 10.1021/ja047274x

[9] ROBINSON, J. T. ; TABAKMAN, S. M. ; LIANG, Y. ; WANG, H. ; SNACHEZ CASALONGUE, H. ; VINH, D. ; DAI, H.: Ultrasmall reduced graphene oxide with high near-infrared absorbance for photothermal therapy. J. Am. Chem. Soc. 133 (2011), pp. 68256831; DOI: 10.1021/ja2010175

[10] HUMMERS, W. S. ; OFFEMAN, R. E.: Preparation of graphitic oxide. J. Am. Chem. Soc. 80 (1958), pp. 1339-1339; DOI: 10.1021/ja01539a017

[11] BREUER, L. ; RAUE, M. KIRSCHBAUM, M. ; MANG, T. ; SCHÖNING, M. J. ; THOELEN, R. ; WAGNER, T.: Light-controllable polymeric material based on temperature-sensitive hydrogels with incorporated graphene oxide. Phys. Status Solidi $A$ $212 \quad$ (2015), pp.1368-1374; DOI: 10.1002/pssa.201431944 